\title{
Multiple piezo-patch energy harvesters integrated to a thin plate with AC-DC conversion: Analytical modeling and numerical validation
}

\author{
Amirreza Aghakhani*a, Ipek Basdogan ${ }^{\mathrm{a}}$, Alper Erturk ${ }^{\mathrm{b}}$ \\ ${ }^{a}$ Department of Mechanical Engineering, College of Engineering, \\ Koç University, Istanbul, 34450, Turkey; \\ ${ }^{\mathrm{b}} \mathrm{G}$. W. Woodruff School of Mechanical Engineering, \\ Georgia Institute of Technology, Atlanta, GA, 30332-0405, USA
}

\begin{abstract}
Plate-like components are widely used in numerous automotive, marine, and aerospace applications where they can be employed as host structures for vibration based energy harvesting. Piezoelectric patch harvesters can be easily attached to these structures to convert the vibrational energy to the electrical energy. Power output investigations of these harvesters require accurate models for energy harvesting performance evaluation and optimization. Equivalent circuit modeling of the cantilever-based vibration energy harvesters for estimation of electrical response has been proposed in recent years. However, equivalent circuit formulation and analytical modeling of multiple piezo-patch energy harvesters integrated to thin plates including nonlinear circuits has not been studied. In this study, equivalent circuit model of multiple parallel piezoelectric patch harvesters together with a resistive load is built in electronic circuit simulation software SPICE and voltage frequency response functions (FRFs) are validated using the analytical distributedparameter model. Analytical formulation of the piezoelectric patches in parallel configuration for the DC voltage output is derived while the patches are connected to a standard AC-DC circuit. The analytic model is based on the equivalent load impedance approach for piezoelectric capacitance and AC-DC circuit elements. The analytic results are validated numerically via SPICE simulations. Finally, DC power outputs of the harvesters are computed and compared with the peak power amplitudes in the AC output case.
\end{abstract}

Keywords: Piezoelectricity, energy harvesting, equivalent circuits

\section{INTRODUCTION}

Vibrational energy harvesting has received significant attention over the past decade with the ultimate goal of powering small electronic components, replacing the conventional batteries and thereby enabling self-powered systems ${ }^{1,2}$. Various vibration-to-electricity conversion methods such electrostatic ${ }^{3}$, electromagnetic ${ }^{4}$, magnetostrictive ${ }^{5}$, and piezoelectric transducers ${ }^{6}$, as well as the electroactive and electrostrictive polymers ${ }^{7,8}$ have been used, among which piezoelectric transduction is the most favored one due to the high power density of piezoelectric materials and ease of implementation at different geometric scales ${ }^{9}$.

In the two decades of piezoelectric energy harvesting literature, cantilever beam harvesters have been explored ${ }^{10,11}$ in many studies. Different theoretical models ${ }^{12,13}$ together with numerical ${ }^{14,15}$ and/or experimental verifications ${ }^{16}$ have been presented by different researchers. Among the many harvesting studies in the literature, few studies have focused on energy harvesting from two-dimensional plate-like structures. Piezo-patch energy harvesters attached to a thin plate have the merits of removing extra mass loading and volumetric occupancy as well as exhibiting relatively large number of vibrational modes compared to the conventional cantilever designs. In addition, frequent use of thin plates/shells in aerospace, automotive and marine applications, makes this implementation convenient to employ and provide broadband energy harvesting. Recently, analytical closed-form expressions and experimental validations for AC electrical outputs of multiple piezoelectric patch energy harvesters structurally integrated to a thin plate is presented by Aridogan et al. ${ }^{17}$. The current work extends that model to the AC input - DC output case, where an AC-DC converter circuit is connected to the parallel configurations of the multiple piezo-patch harvesters.

Smart Materials and Nondestructive Evaluation for Energy Systems 2016, edited by

Norbert G. Meyendorf, Theodoros E. Matikas, Kara J. Peters, Proc. of SPIE Vol. 9806, 98060C

· (c) 2016 SPIE · CCC code: 0277-786X/16/\$18 · doi: 10.1117/12.2219079

Proc. of SPIE Vol. 9806 98060C-1 
In the modeling of both cantilever beam and plate-like harvesters, usually an equivalent resistive load is used to predict the AC power output. However, for transferring the electrical harvested power to the practical end devices (e.g. batteries, storage capacitor, etc.), a stable DC signal is required. A standard interface circuit that provides a stable DC output at the resistive load includes a full-wave bride rectifier followed by a smoothing capacitor. Equivalent circuit model of piezopatch energy harvesters with nonlinear circuit elements can be built into an electronic circuit simulator (e.g., SPICE) to predict the electrical outputs. The analytical ${ }^{18,19}$ and numerical ${ }^{15,18}$ methods for obtaining the equivalent circuit parameters of beam harvesters have been presented in the literature. More recently, Bayik et al. ${ }^{20}$ have developed the multi-modal analytical and numerical approach for identifying the equivalent circuit parameters of a single piezo-patch harvester attached on a thin plate. On the other hand, several studies have focused on analytical modeling for estimating the DC voltage. Lien and Shu ${ }^{21}$ used the energy balance analysis together with the equivalent impedance implementation, which represents the harvesting circuit by an equivalent electrical impedance, to estimate the steadystate DC voltage output of the single-degree-of-freedom (SDOF) harvester. They utilized the equivalent impedance method for an array of cantilever beam harvesters connected in parallel.

This paper presents the analytical formulation with numerical validations of DC electrical outputs for the parallel connection of multiple piezo-patch energy harvesters attached on a thin plate. Analytical model for the steady-state DC voltage output is derived based on the equivalent impedance method. Equivalent impedance of the electromechanical parts by accounting for multiple vibration-modes together with the interface circuit are built in the electronic circuit simulator (e.g., SPICE), and simulations are performed for the cases of AC input - AC output and AC input - DC output.

\section{ANALYTICAL DISTRIBUTED-PARAMETER MODELLING}

Figure 1 presents the multiple piezo-patch energy harvesters connected in parallel and attached on a thin plate. The host plate with the length and width of $a$ and $b$ is excited by a transverse point force $f(t)$ acting at the position coordinates $\left(x_{0}, y_{0}\right)$. The plate thickness $\left(h_{\overline{\mathrm{s}}}\right)$ is assumed to be much smaller than other dimensions so that the effects of transverse shear deformation are neglected based on the Kirchhoff plate theory. In the following, the subscripts $\overline{\mathrm{s}}$ and $\overline{\mathrm{p}}$ stand for the parameters related to the host structure and piezo-patches, respectively. The number of piezo-patch harvesters is $n_{\overline{\mathrm{p}}}$, and each piezoceramic patch covers a rectangular area with two corners at $\left(x_{k, 1}, y_{k, 1}\right)$ and $\left(x_{k, 2}, y_{k, 2}\right)$ where $k=1,2, \ldots, n_{\overline{\mathrm{p}}}$. The thickness of the $k$-th patch is $\left(h_{\overline{\mathrm{p}}}\right)_{k}$ and the polarization is in thickness direction.

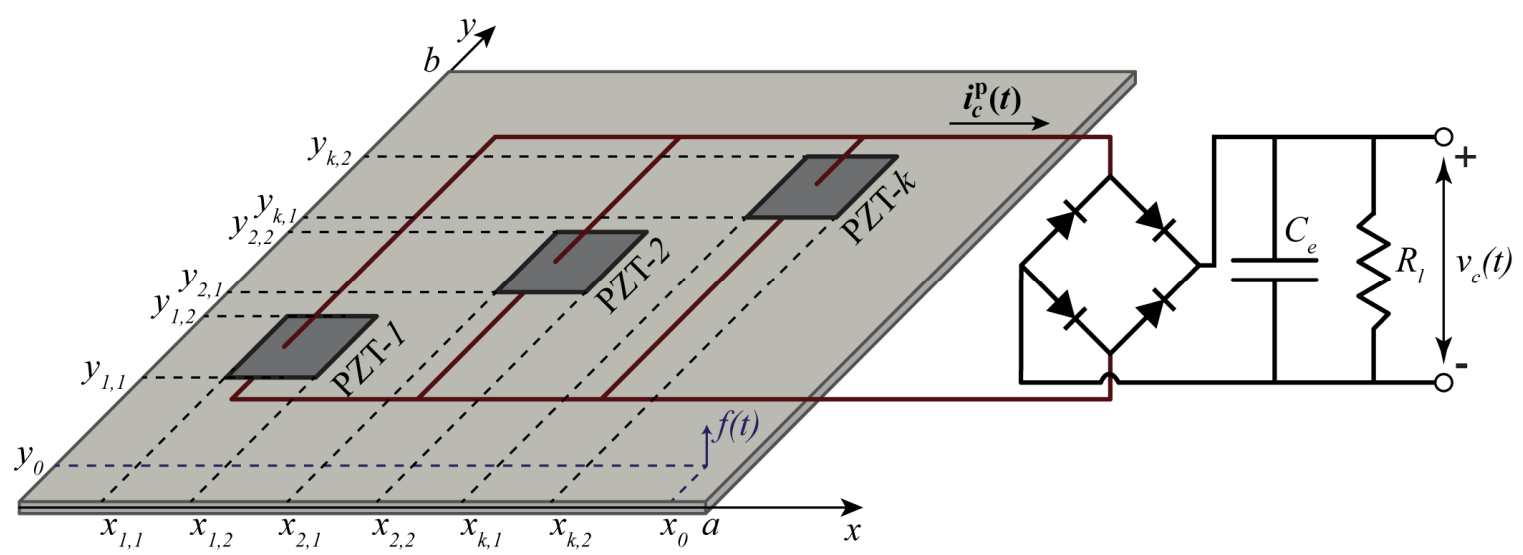

Figure 1. Parallel configuration of Multiple piezo-patch energy harvesters attached on a thin plate with a practical interface circuit

By conducting the modal analysis procedure for the two-dimensional structure ${ }^{17}$, electromechanically coupled ordinary differential equations of the plate in modal coordinates can be written as 


$$
\begin{gathered}
\frac{\mathrm{d}^{2} \eta_{m n}^{\mathrm{p}}(t)}{\mathrm{d} t^{2}}+2 \zeta_{m n} \omega_{m n} \frac{\mathrm{d} \eta_{m n}^{\mathrm{p}}(t)}{\mathrm{d} t}+\omega_{m n}^{2} \eta_{m n}^{\mathrm{p}}(t)-v^{\mathrm{p}}(t) \sum_{k=1}^{n_{\overline{\mathrm{p}}}}\left(\widetilde{\theta}_{m n}\right)_{k}=f_{m n}(t) \\
\sum_{k=1}^{n_{\overline{\mathrm{p}}}} \sum_{n=1}^{\infty} \sum_{m=1}^{\infty}\left(\tilde{\theta}_{m n}\right)_{k} \frac{\mathrm{d} \eta_{m n}^{\mathrm{p}}(t)}{\mathrm{d} t}+\frac{\mathrm{d} v^{\mathrm{p}}(t)}{\mathrm{d} t} \sum_{k=1}^{n_{\overline{\mathrm{p}}}}\left(C_{\overline{\mathrm{p}}}\right)_{k}+i_{c}^{\mathrm{p}}(t)=0
\end{gathered}
$$

where $\eta_{m n}^{\mathrm{p}}(t), \omega_{m n}$ and $\zeta_{m n}$ are the corresponding modal coordinate, undamped natural frequency and modal damping ratio of the plate for the $m n$-th vibration mode. The equivalent capacitance for the $k$-th piezo-patch is defined as $\left(C_{\overline{\mathrm{p}}}\right)_{k}=\left(\bar{\varepsilon}_{33}^{\mathrm{S}}\right)_{k} \frac{\left(l_{\overline{\mathrm{p}}}\right)_{k}\left(w_{\overline{\mathrm{p}}}\right)_{k}}{\left(h_{\overline{\mathrm{p}}}\right)_{k}}$. The piezo-patch dimension terms $\left(l_{\overline{\mathrm{p}}}\right)_{k},\left(w_{\overline{\mathrm{p}}}\right)_{k}$ and $\left(h_{\overline{\mathrm{p}}}\right)_{k}$ are the length, width and thickness of the $k$ th patch harvester, respectively, and $\left(\bar{\varepsilon}_{33}^{\mathrm{S}}\right)_{k}$ is the permittivity of the $k$ th patch at constant strain. The electromechanical coupling term $\left(\widetilde{\theta}_{m n}\right)_{k}$ and modal forcing input $f_{m n}(t)$ can be given by

$$
\begin{gathered}
\left(\tilde{\theta}_{m n}\right)_{k}=\theta_{k} \int_{y_{k, 1}}^{y_{k, 2}} \int_{x_{k, 1}}^{x_{k, 2}}\left[\frac{\partial^{2} \phi_{m n}(x, y)}{\partial x^{2}}+\frac{\partial^{2} \phi_{m n}(x, y)}{\partial y^{2}}\right] \mathrm{d} x \mathrm{~d} y \\
f_{m n}(t)=f(t) \phi_{m n}\left(x_{0}, y_{0}\right)
\end{gathered}
$$

Here, $\phi_{m n}(x, y)$ is the mass-normalized eigenfunction of the plate for the $m n$-th vibration mode at $(x, y)$ location. The electromechanical term $\theta_{k}$ for the $k$ th patch harvester is defined as $\theta_{k}=\left(h_{\mathrm{pc}}\right)_{k}\left(\bar{e}_{31}\right)_{k}$, which is the multiplication of effective piezoelectric constant $\left(\bar{e}_{31}\right)_{k}$ and reference distance $\left(h_{\mathrm{pc}}\right)_{k}$ of the center layer of $k$ th piezo-patch from the reference surface (i.e., the neutral surface level of the plate in the piezo region) at the location of the $k$-th patch. Note that the voltage across each piezo electrode is equal and given by $v^{\mathrm{p}}=v_{k}^{\mathrm{p}}(t)$, and the current flowing to the interface circuit is identified by $i_{c}^{\mathrm{p}}(t)$.

\section{EQUIVALENT IMPEDANCE MODELING}

\subsection{Equivalent Impedance of the mechanical domain}

The analytical distributed-parameter model of the plate with multiple piezo-patch energy harvesters is presented for the $\mathrm{AC}$ input - AC output case, where the interface circuit is a simple resistive load ${ }^{17}$. However, in practical applications where the storage systems usually require a stable DC signal, a standard full-wave rectifier circuit is employed for ACDC conversion. With presence of nonlinear circuit elements, such as diodes, one approach to estimate the DC power output is to build the equivalent circuit model of the electromechanical system in the electronic software simulation (e.g., SPICE) and perform transient simulation. The presented model in this study, accounts for two-way electromechanical coupling and multi-vibration mode of the structure.

Figure 2 demonstrates the equivalent circuit modeling for the multiple piezo-patch harvesters connected in parallel. Each vibrational mode of the harvester is represented as a second-order circuit connected to each piezo-patch capacitance $\left(\left(C_{\overline{\mathrm{p}}}\right)_{k}\right)$. By applying Kirchhoff's voltage law to the multi-vibration mode circuit and by analogy with equation (1), system parameters of the equivalent circuit model can be determined. Table 1 gives a summary of the analogy between the analytical distributed-parameter expression and the equivalent circuit model. 


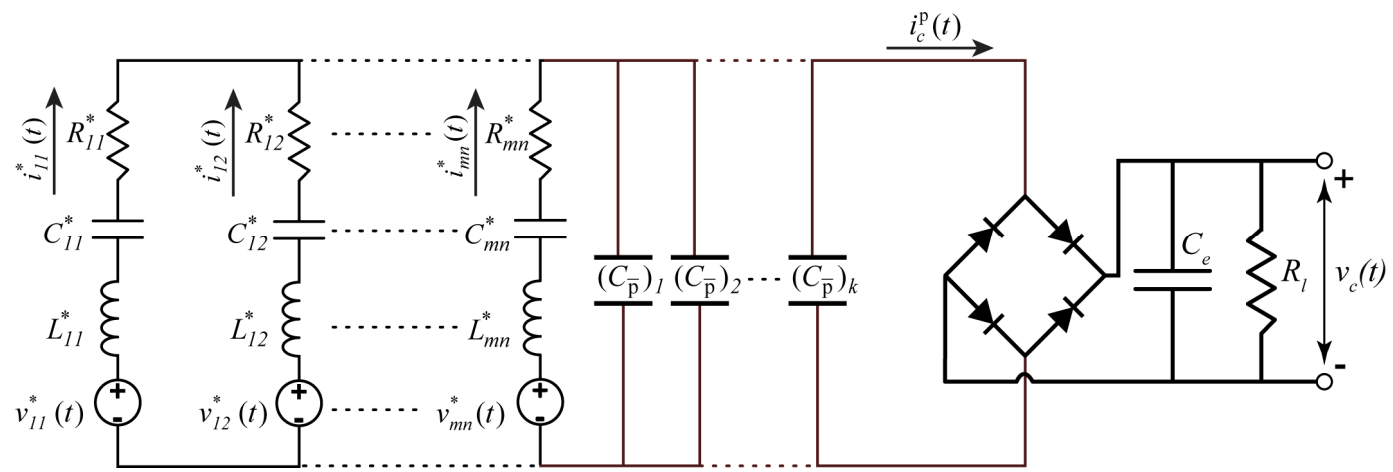

Figure 2. Equivalent circuit representation of the multiple piezo-patch harvesters (parallel configuration) connected to an AC-DC converter by accounting for multiple vibration modes (a total of $m \times n$ modes).

Table 1. Analogy between electrical and mechanical domains of multiple piezo-patch energy harvesters integrated to a thin plate

\begin{tabular}{|l|l|}
\hline Equivalent circuit parameters & Mechanical counterparts \\
\hline Voltage source: $v_{m n}^{*}(t)$ & $-\left(\sum_{k=1}^{n_{\overline{\mathrm{p}}}}\left(\widetilde{\theta}_{m n}\right)_{k}\right)^{-1} f_{m n}(t)$ \\
\hline Electrical current: $i_{m n}^{*}(t)$ & $-\sum_{k=1}^{n_{\overline{\mathrm{p}}}}\left(\widetilde{\theta}_{m n}\right)_{k} \dot{\eta}_{m n}^{\mathrm{p}}(t)$ \\
\hline Inductance: $L_{m n}^{*}$ & $\left(\sum_{k=1}^{n_{\overline{\mathrm{p}}}}\left(\tilde{\theta}_{m n}\right)_{k}\right)^{-2}$ \\
\hline Resistance: $R_{m n}^{*}$ & $2 \zeta_{m n} \omega_{m n}\left(\sum_{k=1}^{n_{\overline{\mathrm{p}}}}\left(\widetilde{\theta}_{m n}\right)_{k}\right)^{-2}$ \\
\hline Capacitance: $C_{m n}^{*}$ & $\omega_{m n}{ }^{-2}\left(\sum_{k=1}^{n_{\overline{\mathrm{p}}}}\left(\widetilde{\theta}_{m n}\right)_{k}\right)^{2}$ \\
\hline
\end{tabular}

\subsection{Equivalent impedance of the electrical domain}

In this section, using the energy balance method and equivalent impedance of the electrical circuit, explicit analytical solutions for steady-state DC voltage and power outputs are derived. For modeling of the AC-DC converter circuit, it's been assumed that the diodes are ideal, thus their voltage drop is zero. Additionally, the current flowing to the AC-DC converter circuit $i_{c}^{\mathrm{p}}(t)$ as shown in figure 1 can be described by the following piecewise equation: 


$$
i_{c}^{\mathrm{p}}(t)= \begin{cases}C_{e} \dot{v}_{c}(t)+\frac{v_{c}(t)}{R_{l}} & \text { if } v^{\mathrm{p}}=v_{c}^{\mathrm{p}} \\ -C_{e} \dot{v}_{c}(t)-\frac{v_{c}(t)}{R_{l}} & \text { if } v^{\mathrm{p}}=-v_{c}^{\mathrm{p}} \\ 0 & \text { if }\left|v^{\mathrm{p}}\right|<v_{c}^{\mathrm{p}}\end{cases}
$$

where $v_{c}^{\mathrm{p}}(t)$ is the rectified voltage over the load resistance. If the smoothing capacitor is carefully chosen so that the output time constant $R_{l} C_{e}$ is much larger than the period of the excitation, the DC voltage output will have a constant $V_{c}^{\mathrm{p}}$ magnitude, and the ripple in the rectified voltage will be negligible for large values of smoothing capacitor ${ }^{22,23}$. The steady-state solution for the point force excitation in the form of $f(t)=F_{0} e^{\mathrm{j} \omega t}$ can be obtained by setting the modal coordinate as

$$
\eta_{m n}^{\mathrm{p}}(t)=\mathrm{H}_{m n}^{\mathrm{p}} e^{\mathrm{j}\left(\omega t+\tau_{m n}\right)}
$$

where $\mathrm{H}_{m n}^{\mathrm{p}}$ is the complex amplitude for the modal coordinate and $\tau_{m n}$ is the unknown phase shift between the force excitation and the vibration response in modal coordinate. From the current balance equation in (2), the equivalent current source of the harvester can be defined as

$$
i^{\mathrm{p}}(t)=\sum_{k=1}^{n_{\overline{\mathrm{p}}}} \sum_{n=1}^{\infty} \sum_{m=1}^{\infty}\left(i_{m n}^{\mathrm{p}}(t)\right)_{k},\left(i_{m n}^{\mathrm{p}}(t)\right)_{k}=-\left(\tilde{\theta}_{m n}\right)_{k} \frac{\mathrm{d} \eta_{m n}^{\mathrm{p}}(t)}{\mathrm{d} t}
$$

Further, the dependent current source $i_{k}^{\mathrm{p}}(t)$ is essentially summation of the electrical current $\left(i_{m n}^{\mathrm{p}}(t)\right)_{k}$ over all the $m$ by $n$ modes of the $k$-th piezo-patch harvester and its complex amplitude can be written as

$$
I_{k}^{\mathrm{p}}=\sum_{n=1}^{\infty} \sum_{m=1}^{\infty}\left(I_{m n}\right)_{k},\left(I_{m n}\right)_{k}=-j \omega\left(\tilde{\theta}_{m n}\right)_{k} \mathrm{H}_{m n}^{\mathrm{p}} e^{\mathrm{j} \tau_{m n}}
$$

Finally, steady-state form of the overall current source $i^{\mathrm{p}}(t)$ is given by

$$
i^{\mathrm{p}}(t)=I^{\mathrm{p}} e^{j \omega t}, I^{\mathrm{p}}=\sum_{k=1}^{n_{\overline{\mathrm{p}}}} I_{k}^{\mathrm{p}}
$$

Integrating the current balance equation in (2) over the half-cycle period of the excitation, while substituting the current flown to the AC-DC circuit with (5) gives

$$
V_{c}^{\mathrm{p}}=\left(\frac{R_{l}}{\frac{\pi}{2}+\left(R_{l} \omega\right) C_{\overline{\mathrm{p}}}^{e q}}\right) I^{\mathrm{p}}
$$

Here, $C_{\overline{\mathrm{p}}}^{e q}$ is the overall piezoelectric capacitance for the parallel connection of piezo-patches and equals to $C_{\overline{\mathrm{p}}}^{e q}=\sum_{k=1}^{n_{\overline{\mathrm{p}}}}\left(C_{\overline{\mathrm{p}}}\right)_{k}$. Now if all the $I_{k}^{\mathrm{p}}$ for $k=1,2, \ldots, n_{\overline{\mathrm{p}}}$ are found, the steady-state DC voltage output for the parallel configuration of multiple piezo-patch harvesters can be directly obtained from (10). To do so, let the $Z_{e q}^{\mathrm{p}}$ be the equivalent impedance for the parallel configuration, which represents the AC-DC circuit dynamics and the equivalent 
piezo capacitance as illustrated in figure 3. Note that the equivalent impedance $Z_{e q}^{\mathrm{p}}$ is the function of excitation frequency, equivalent piezoelectric capacitor and dynamics of the interface circuit. However, it is doesn't depend on the number of vibrational modes and consequently the number of current sources. Determination of the equivalent impedance for a SDOF piezoelectric energy harvester is presented in ${ }^{21}$ where $Z_{e q}^{\mathrm{p}}=Z_{R}^{\mathrm{p}}+j Z_{I}^{\mathrm{p}}$ and its real and imaginary parts are given as

$$
Z_{R}^{\mathrm{p}}=\frac{2 R}{\left(\pi / 2+C_{\overline{\mathrm{p}}}^{e q} R \omega\right)^{2}}, Z_{I}^{\mathrm{p}}=-\frac{R}{\left(\pi / 2+C_{\overline{\mathrm{p}}}^{e q} R \omega\right)}
$$

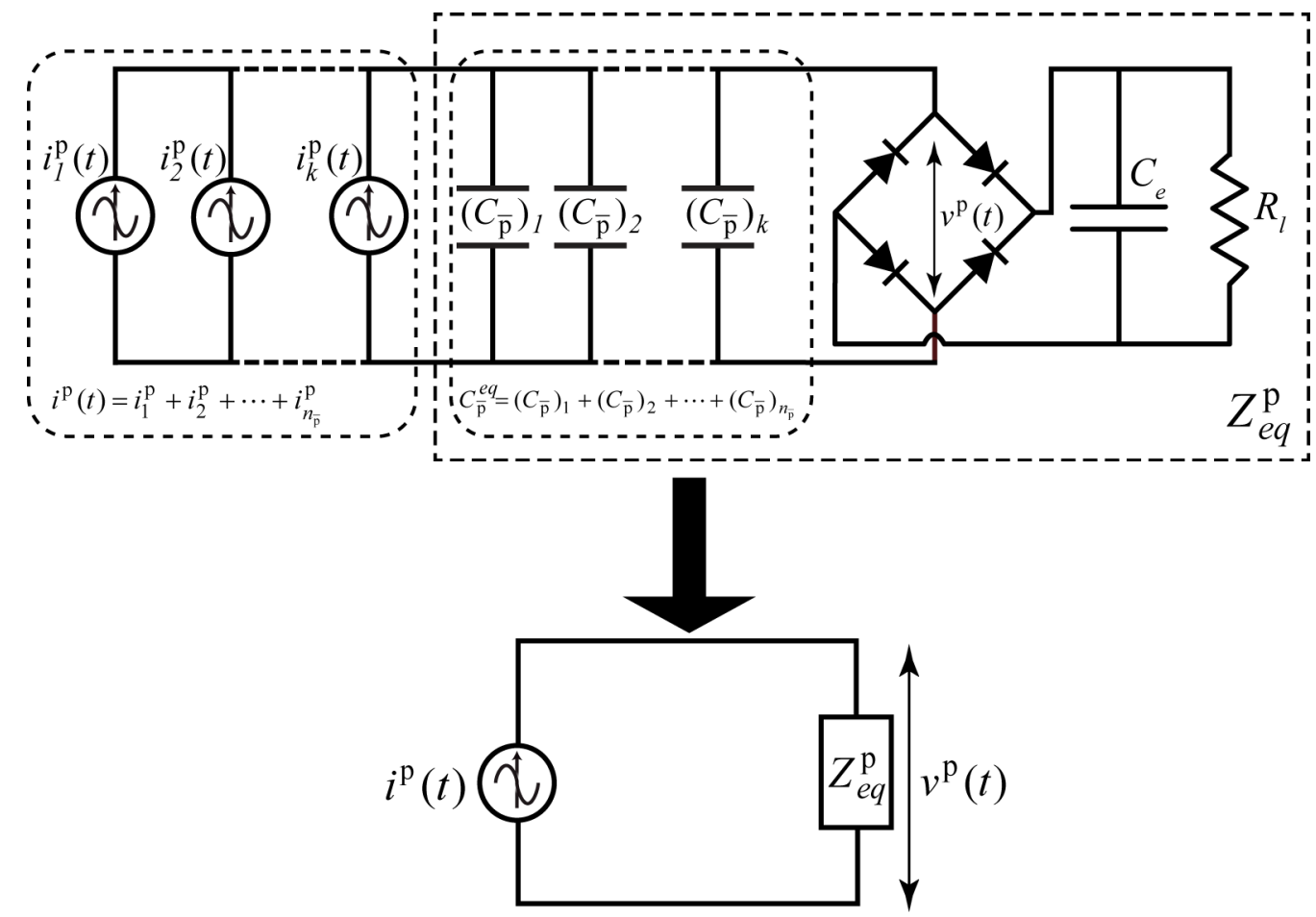

Figure 3. Electrical current model of the harvester: (a) connected AC-DC converter (b) Equivalent Impedance replacing the piezoelectric capacitance and the AC-DC converter

This implementation is valid, since $Z_{e q}^{\mathrm{p}}$ is not related to the mechanical part. The relation of current and voltage passing through $Z_{e q}^{\mathrm{p}}$ in figure 3 can be written as

$$
v^{\mathrm{p}}(t)=Z_{e q}^{\mathrm{p}}\left(i^{\mathrm{p}}(t)\right)=Z_{e q}^{\mathrm{p}}\left(\sum_{k=1}^{n_{\overline{\mathrm{p}}}} i_{k}^{\mathrm{p}}(t)\right)
$$

Replacing the $v^{\mathrm{p}}(t)$ in the equation (1) with (12), the electromechanical equation for the parallel configuration of piezopatches yield to the following steady-state forms

$$
\left(\omega_{m n}^{2}-\omega^{2}+\mathrm{j} 2 \zeta_{m n} \omega_{m n} \omega\right) \mathrm{H}_{m n}^{\mathrm{p}} e^{j \tau_{m n}}-Z_{e q}^{\mathrm{p}}\left(\sum_{k=1}^{n_{\overline{\mathrm{p}}}} I_{k}^{\mathrm{p}}\right) \sum_{k=1}^{n_{\overline{\mathrm{p}}}}\left(\tilde{\theta}_{m n}\right)_{k}=F_{0} \phi_{m n}\left(x_{0}, y_{0}\right)
$$


Dividing the both sides by $\sum_{k=1}^{n_{\overline{\mathrm{p}}}}\left(\widetilde{\theta}_{m n}\right)_{k}$ and rearranging the above equation using (8), we have

$$
\left[\frac{2 \zeta_{m n} \omega_{m n}}{\left(\sum_{k=1}^{n_{\overline{\mathrm{p}}}}\left(\widetilde{\theta}_{m n}\right)_{k}\right)^{2}}+j \frac{\omega^{2}-\omega_{m n}^{2}}{\omega\left(\sum_{k=1}^{n_{\overline{\mathrm{p}}}}\left(\widetilde{\theta}_{m n}\right)_{k}\right)^{2}}\right]\left(\sum_{k=1}^{n_{\overline{\mathrm{p}}}}\left(I_{m n}\right)_{k}\right)+Z_{e q}^{\mathrm{p}}\left(\sum_{k=1}^{n_{\overline{\mathrm{p}}}} \sum_{n=1}^{\infty} \sum_{m=1}^{\infty}\left(I_{m n}\right)_{k}\right)=-\frac{F_{0} \phi_{m n}\left(x_{0}, y_{0}\right)}{\sum_{k=1}^{n_{\overline{\mathrm{p}}}}\left(\widetilde{\theta}_{m n}\right)_{k}}
$$

By defining $I_{m n}=\sum_{k=1}^{n_{\overline{\bar{p}}}}\left(I_{m n}\right)_{k}$ and $V_{m n}=-\frac{F_{0} \phi_{m n}\left(x_{0}, y_{0}\right)}{\sum_{k=1}^{n_{\bar{\Gamma}}}\left(\tilde{\theta}_{m n}\right)_{k}}$, equation (14) can be written in a following matrix equation

$$
\mathbf{V}=\mathbf{Z} \mathbf{I}
$$

where the equivalent voltage amplitude vector $\mathbf{V}$ with the dimensions of $m n \times 1$, equals to $\mathbf{V}=\left[\begin{array}{llll}V_{11} & V_{12} & \cdots & V_{m n}\end{array}\right]^{T}$ and the equivalent current amplitude vector with the dimensions $m n \times 1$ is $\mathbf{I}=\left[\begin{array}{llll}I_{11} & I_{12} & \cdots & I_{m n}\end{array}\right]^{T}$. The square matrix $\mathbf{Z}$ with the dimensions of $m n \times m n$ can be written as

$$
Z_{i j}= \begin{cases}\frac{2 \zeta_{m n} \omega_{m n}}{\left(\sum_{k=1}^{n_{\overline{\mathrm{p}}}}\left(\widetilde{\theta}_{m n}\right)_{k}\right)^{2}}+j \frac{\omega^{2}-\omega_{m n}^{2}}{\omega\left(\sum_{k=1}^{n_{\overline{\mathrm{p}}}}\left(\widetilde{\theta}_{m n}\right)_{k}\right)^{2}}+Z_{e q}^{\mathrm{p}} & i=j \\ Z_{e q}^{\mathrm{p}} & i \neq j\end{cases}
$$

Note that from equation (16), $I^{\mathrm{p}}=\sum_{k=1}^{n_{\overline{\mathrm{p}}}} \sum_{n=1}^{\infty} \sum_{m=1}^{\infty}\left(I_{m n}\right)_{k}=\sum_{n=1}^{\infty} \sum_{m=1}^{\infty} I_{m n}$ and each $I_{m n}$ can be found by solving the linear system of equations in (22). Therefore, the steady-state magnitude of DC voltage across the resistive load can be obtained as

$$
V_{c}=\left(\frac{R_{l}}{\frac{\pi}{2}+\left(R_{l} \omega\right) C_{\overline{\mathrm{p}}}^{e q}}\right)\left|I^{\mathrm{p}}\right|=\left(\frac{R_{l}}{\frac{\pi}{2}+\left(R_{l} \omega\right) C_{\overline{\mathrm{p}}}^{e q}}\right)\left|\sum_{n=1}^{\infty} \sum_{m=1}^{\infty} I_{m n}^{*}\right|
$$

\section{MODEL VALIDATION}

In this section, equivalent circuit model of a fully clamped plate with two perfectly bonded piezo-patches is constructed in SPICE software and simulation results are validated for the standard AC input - AC output and AC input - DC output problems. Equivalent circuit model results for $\mathrm{AC}$ output configuration are validated using the distributed parameter model developed by Aridogan et al ${ }^{17}$. For DC output configuration, the closed-form analytical expression (in section 3 ) is used for obtaining the DC output results and compared with numerical simulations. The geometric and material properties of the aluminum plate and piezo-patches are given in Table 2. 
Table 2. Geometric, material and dielectric properties

\begin{tabular}{|l|c|c|}
\hline Property & Aluminum & Piezoceramic \\
\hline Length $(\mathrm{mm})$ & 580 & 72.4 \\
\hline Width $(\mathrm{mm})$ & 540 & 72.4 \\
\hline Thickness $(\mathrm{mm})$ & 1.96 & 0.267 \\
\hline Young's modulus $(\mathrm{GPa})$ & 70 & 66 \\
\hline Mass density $\left(\mathrm{kg} \mathrm{m}^{-3}\right)$ & 2700 & 7800 \\
\hline Piezoelectric constant $d^{31}\left(\mathrm{pm} \mathrm{V}^{-1}\right)$ & - & -190 \\
\hline Permittivity constant $\bar{\varepsilon}_{33}^{S}\left(\mathrm{nF} \mathrm{m}^{-1}\right)$ & - & 10.38 \\
\hline Damping ratio $\zeta_{m n}$ & 0.01 & 0.01 \\
\hline
\end{tabular}

\subsection{AC input - AC output case study}

In the standard $\mathrm{AC}$ input - $\mathrm{AC}$ output problem, the energy harvesting circuit is simply represented by only a resistance load. Parameters of the multi-mode equivalent circuit model in Table 1 are identified from analytical model considering the $5 \times 5$ vibration modes of the plate. Steady-state voltage frequency response functions (FRFs) of the multiple piezopatch harvesters in parallel configuration under the effect of $1 \mathrm{~N}$ harmonic point force are shown for the first four vibration modes in figure 4 with resistive load cases ranging from $100 \Omega$ to $1 \mathrm{M} \Omega$. The first four short-circuit resonance frequencies of the fully clamped plate in this case study are 53.32, 103.90, 183.84 and $206.54 \mathrm{~Hz}$.
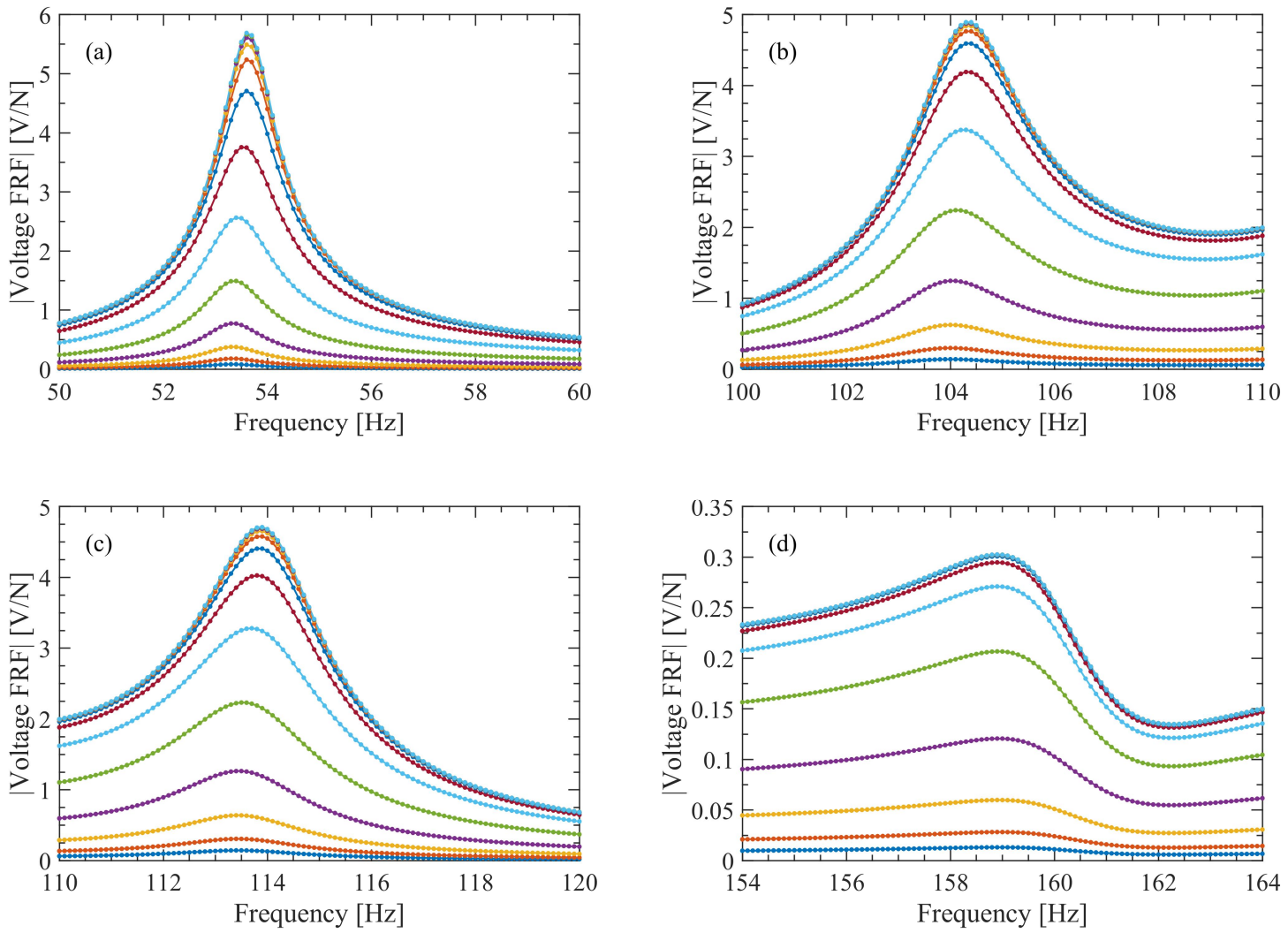

Figure 4. AC input - AC output case: Comparison of analytical (solid line) and SPICE (dot markers) voltage FRFs for the multiple piezo-patch harvesters connected in parallel: a) first mode b) second mode c) third mode d) fourth mode 


\subsection{AC input - DC output case study}

The standard AC-DC piezoelectric energy harvesting circuit consists of a full-wave rectifier, a smoothing capacitor, and a resistive load as shown in figure 1. Derivation of the analytical model for estimating the DC voltage output of multiple piezo-patch harvesters was presented in section 3 assuming ideal behavior of the diodes. Transient simulations over wide range of resistance values are performed to estimate the steady-state DC voltage across the load resistance. Note that since the unwanted ripple in the voltage and current output depend on the output time constant $R_{l} C_{e}$, smoothing capacitor is carefully chosen so that that the product of $R_{l} C_{e}$ is much higher than the ripple period. To this end, in all simulations to ensure the stable voltage output, the output time constant is always around 0.1 seconds, which is approximately ten times the period of voltage/current ripple at the first vibration mode. Therefore, for the load resistance values ranging from $100 \Omega$ to $1 \mathrm{M} \Omega$, the smoothing capacitor values differ from $1 \mathrm{mF}$ to $100 \mathrm{nF}$.

Figure 5 presents the comparison of SPICE simulation and the analytical results for DC voltage FRFs across the various load resistances $(100 \Omega$ to $1 \mathrm{M} \Omega)$ under $1 \mathrm{~N}$ harmonic force excitation. Results again are shown for the first four vibration modes of the structure.
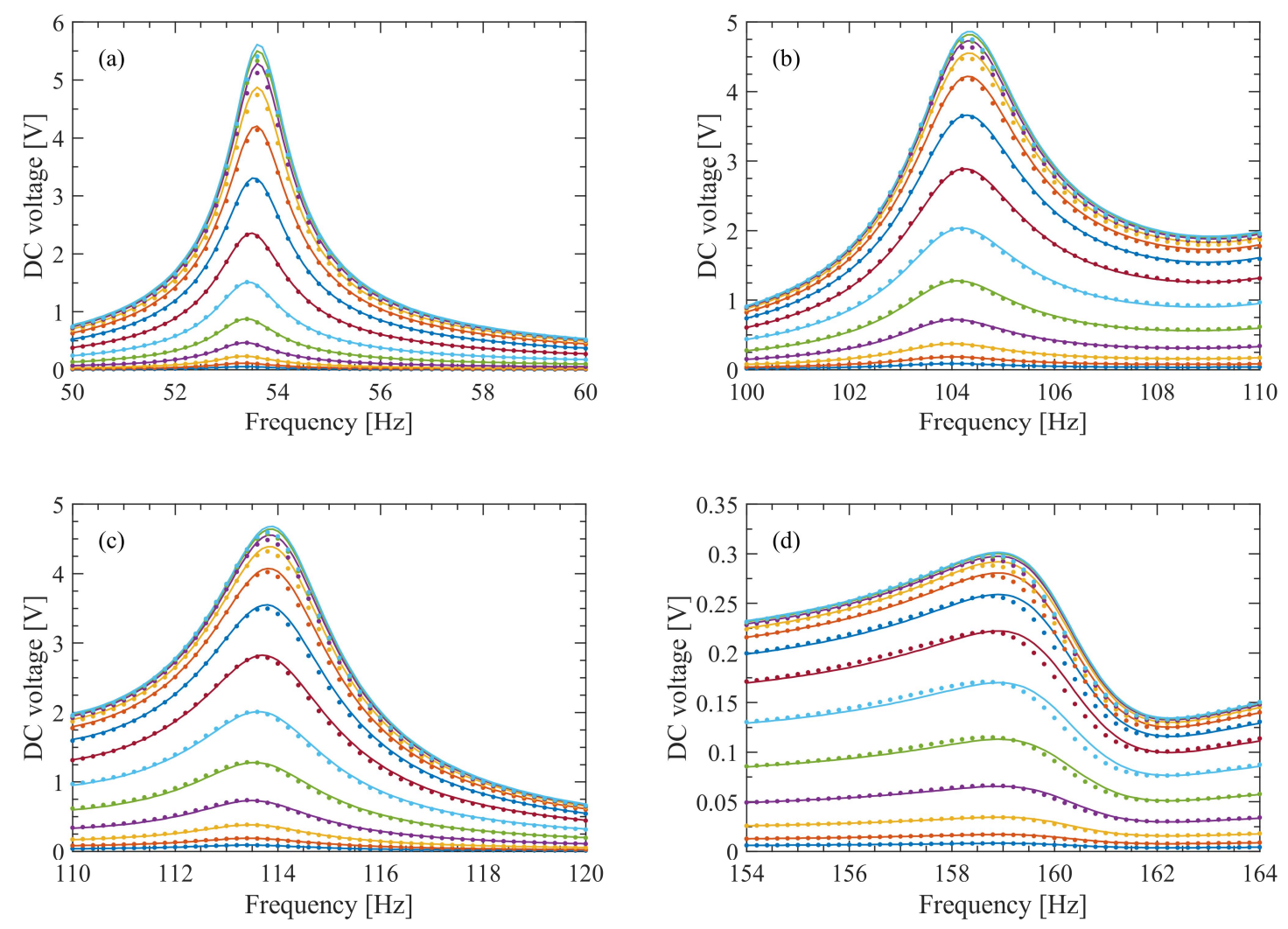

Figure 5. AC input - DC output case: Comparison of the analytically derived model (solid lines) and SPICE simulation results (dot markers) for the parallel configuration of multiple piezo-patch harvesters: (a) first mode, (b) second mode (c) third mode d) fourth mode

\subsection{Comparison of power outputs for AC input - AC output \& AC input - DC output problems}

To investigate the variation of electrically harvested power with different interface circuits, analytical and numerical simulations of peak power output with different resistance load values, for the first four vibration modes, are presented 
in figure 6. Power peak amplitudes are extracted at the open-circuit resonance frequency of the plate under $1 \mathrm{~N}$ excitation force input. The analytical and SPICE simulation results for the modal power outputs are in perfect agreement for both cases.

The power curves have similar trends for both AC - AC and AC - DC cases, exhibiting an optimum resistive load for the maximum power output. Note that in both cases, strain mode shape related cancellation can be seen for the fourth vibration mode. It is also clear that in the $\mathrm{AC}-\mathrm{AC}$ case, more power is delivered to the electrical part compared to the $\mathrm{AC}-\mathrm{DC}$ case. The reason can be referred to the general impedance matching theory ${ }^{24}$, that in weakly coupled system like the plate harvester investigated here, the equivalent resistance in the mechanical impedance (as given in Table 1) is usually much larger than the real component of the equivalent electrical impedance $Z_{e q}^{\mathrm{p}}$. Therefore, with presence of the AC-DC converter, magnitude of $Z_{R}^{\mathrm{p}}$ is usually lower than the value of simple resistance load $R_{l}$ in the $\mathrm{AC}-\mathrm{AC}$ case, which causes less power to flow into the harvesting circuit.
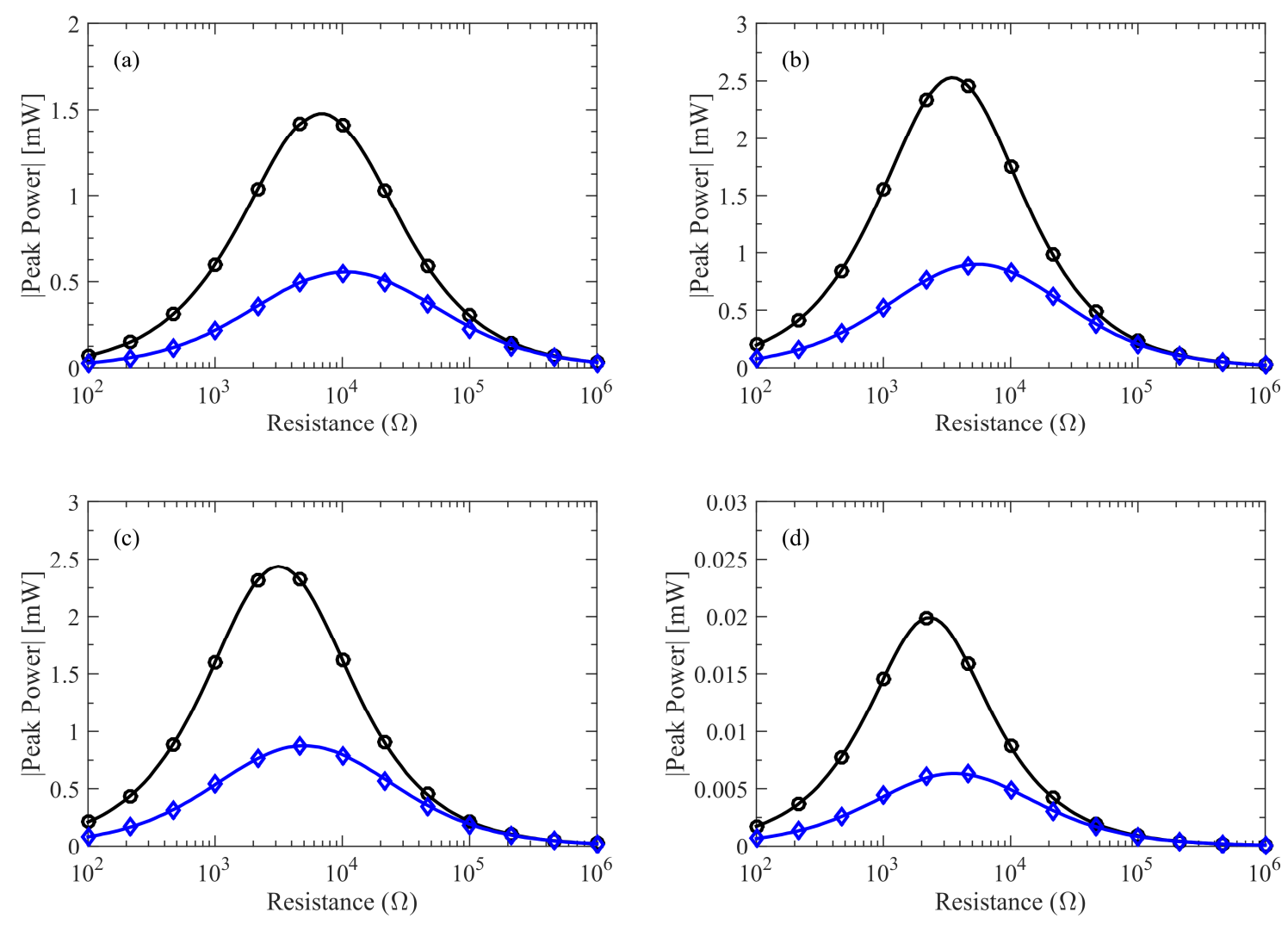

Figure 6.Variations of the peak power amplitudes with load resistance at the open-circuit resonance frequencies for the AC input - AC output (in black) and AC input - DC output (in blue) cases: a) first mode b) second mode c) third mode d) fourth mode. Analytical models shown with solid lines and numerical simulation shown with markers.

\section{CONCLUSION}

Multiple piezo-patch harvesters are considered as an alternative to cantilevered beam harvesters for exploiting the structural vibrations of thin plates without modifying the dynamics. In this study, closed-form the analytical expression was derived for modeling DC electrical outputs of the harvesters connected in parallel. The equivalent circuit model of the electromechanical system was developed and constructed in SPICE software, and simulation results of voltage frequency response functions were validated against the existing analytical model ${ }^{17}$ in the AC input - AC output case. 
Then, using the multi-mode equivalent circuit parameters, transient simulations were conducted in the AC input - DC output case for wide range of resistance load values. Steady-state DC electrical responses were investigated for the first four modes of the plate. Resonant DC power outputs across different resistive loads were computed and compared with the peak voltage amplitudes in the AC output case. The multi-mode equivalent circuit model as well as the equivalent impedance approach proposed in this study enable exploring harvesting performance of the single/multiple piezo-patch harvesters attached to thin plates with any linear and nonlinear electrical components.

\section{ACKNOWLEDGMENTS}

Support from the Koç University TÜPRAŞ Energy Center (KÜTEM) is gratefully acknowledged.

\section{REFERENCES}

[1] Erturk, A. and Inman, D. J., [Piezoelectric Energy Harvesting], John Wiley \& Sons, Ltd, Chichester (2011).

[2] Elvin, N. and Erturk, A., [Advances in energy harvesting methods], Springer Science \& Business Media (2013).

[3] Torres, E. O. and Rincón-Mora, G. A., "A 0.7- $\mu \mathrm{m}$ biCMOS electrostatic energy-harvesting system IC," IEEE Journal of Solid-State Circuits 45(2), 483-496 (2010)

[4] Beeby, S. P., Torah, R. N., Tudor, M. J., Glynne-Jones, P., O'Donnell, T., Saha, C. R. and Roy, S., "A micro electromagnetic generator for vibration energy harvesting," Journal of Micromechanics and Microengineering 17(7), 1257-1265 (2007)

[5] Wang, L. and Yuan, F. G., "Vibration energy harvesting by magnetostrictive material," Smart Materials and Structures 17(4), 045009 (2008)

[6] Roundy, S. and Wright, P. K., "A piezoelectric vibration based generator for wireless electronics," Smart Materials and Structures 13(5), 1131 (2004)

[7] Ren, K., Liu, Y., Hofmann, H., Zhang, Q. M. and Blottman, J., "An active energy harvesting scheme with an electroactive polymer," Applied Physics Letters 91(13), 132910 (2007)

[8] Yiming, L., Kai Liang, R., Hofmann, H. F. and Qiming, Z., "Investigation of electrostrictive polymers for energy harvesting," Ultrasonics, Ferroelectrics, and Frequency Control, IEEE Transactions on 52(12), 2411-2417 (2005)

[9] Cook-Chennault, K., Thambi, N. and Sastry, A., "Powering MEMS portable devices-a review of non-regenerative and regenerative power supply systems with special emphasis on piezoelectric energy harvesting systems," Smart Materials and Structures 17(4), 043001 (2008)

[10] Anton, S. R. and Sodano, H. A., "A review of power harvesting using piezoelectric materials (2003-2006)," Smart Materials and Structures 16(3), R1-R21 (2007)

[11]Friswell, M. I. and Adhikari, S., "Sensor shape design for piezoelectric cantilever beams to harvest vibration energy," Journal of Applied Physics 108(1), 014901 (2010)

[12] Erturk, A. and Inman, D. J., "A distributed parameter electromechanical model for cantilevered piezoelectric energy harvesters," Journal of Vibration and Acoustics 130(4), 041002 (2008)

[13]Erturk, A., "Assumed-modes modeling of piezoelectric energy harvesters: Euler-Bernoulli, Rayleigh, and Timoshenko models with axial deformations," Computers \& Structures 106-107, 214-227 (2012)

[14] Yang, Y. and Tang, L., "Equivalent Circuit Modeling of Piezoelectric Energy Harvesters," Journal of Intelligent Material Systems and Structures 20(18), 2223-2235 (2009)

[15]Elvin, N. G. and Elvin, A. A., "A coupled finite element-circuit simulation model for analyzing piezoelectric energy generators," Journal of Intelligent Material Systems and Structures 20(5), 587-595 (2009) 
[16] Erturk, A. and Inman, D. J., "An experimentally validated bimorph cantilever model for piezoelectric energy harvesting from base excitations," Smart Materials and Structures 18(2), 025009 (2009)

[17] Aridogan, U., Basdogan, I. and Erturk, A., "Multiple patch-based broadband piezoelectric energy harvesting on plate-based structures," Journal of Intelligent Material Systems and Structures 25(14), 1664-1680 (2014)

[18] Yang, Y. and Tang, L., "Equivalent circuit modeling of piezoelectric energy harvesters," Journal of Intelligent Material Systems and Structures 20(18), 2223-2235 (2009)

[19] Elvin, N. G. and Elvin, A. A., "A general equivalent circuit model for piezoelectric generators," Journal of Intelligent Material Systems and Structures 20(1), 3-9 (2009)

[20] Bayik, B., Aghakhani, A., Basdogan, I. and Erturk, A., "Equivalent circuit modeling of a piezo-patch energy harvester on a thin plate with AC-DC conversion " Smart Materials and Structures, in press (2016)

[21] Lien, I. C. and Shu, Y. C., "Array of piezoelectric energy harvesting by the equivalent impedance approach," Smart Materials and Structures 21(8), 082001 (2012)

[22] Shu, Y. and Lien, I., "Analysis of power output for piezoelectric energy harvesting systems," Smart materials and structures 15(6), 1499 (2006)

[23] Guyomar, D., Badel, A., Lefeuvre, E. and Richard, C., "Toward energy harvesting using active materials and conversion improvement by nonlinear processing," Ultrasonics, Ferroelectrics, and Frequency Control, IEEE Transactions on 52(4), 584-595 (2005)

[24] Junrui, L. and Wei-Hsin, L., "Impedance Modeling and Analysis for Piezoelectric Energy Harvesting Systems," Mechatronics, IEEE/ASME Transactions on 17(6), 1145-1157 (2012) 